Review Article

\title{
Network Meta-Analysis of the Safety of Drug Therapy for Cardiogenic Shock
}

\author{
Xianyong Liao, Lin Qian, Song Zhang $\mathbb{D}^{D}$, Xiang Chen, and Jing Lei \\ Hospital of Chengdu University of Traditional Chinese Medicine, Chengdu, China \\ Correspondence should be addressed to Song Zhang; zhangyuer2000@cdutcm.edu.cn
}

Received 2 May 2020; Revised 2 June 2020; Accepted 1 July 2020; Published 1 August 2020

Academic Editor: Jiafeng Yao

Copyright (c) 2020 Xianyong Liao et al. This is an open access article distributed under the Creative Commons Attribution License, which permits unrestricted use, distribution, and reproduction in any medium, provided the original work is properly cited.

Objectives. (1) To conduct a network meta-analysis of clinical drugs used for cardiogenic shock and (2) provide evidence for the selection of medication for the treatment of this condition. Methods. PubMed, EMBASE, Cochrane library, China HowNet (CNKI), Wanfang database, and Weipu database were searched using keywords Dopamine, Dobutamine, Epinephrine, Adrenaline, Norepinephrine, Noradrenaline, Milrinone, Natriuretic peptide, Recombinant human brain natriuretic peptide, Levosimendan, Cardiac shock, and Cardiogenic shock. We select literature according to prespecified inclusion and exclusion criteria and record data such as drug type, mortality, and adverse reactions. Results. Twenty-eight of 1387 articles met inclusion criteria, comprising 1806 patients who suffered from cardiogenic shock. Dopamine, dobutamine, epinephrine, norepinephrine, milrinone, recombinant human brain natriuretic peptide, and levosimendan were all commonly used in the treatment of cardiogenic shock. Milrinone was most effective at reducing mortality and had the lowest incidence of adverse reactions. Conclusion. This network meta-analysis demonstrated that milrinone was the most effective medication at reducing mortality and adverse events in patients suffering from cardiogenic shock.

\section{Introduction}

Cardiogenic shock is characterized by a decline in cardiac function leading to a significant decrease in cardiac output and insufficient effective circulating blood volume, resulting in severe acute peripheral circulatory failure. The mortality rate from cardiogenic shock ranges from $50 \%$ to $80 \%$ [1]. The most common cause of cardiogenic shock is acute myocardial infarction (AMI), accounting for $80 \%$ of cases [2]. Approximately $50 \%$ of patients with AMI develop cardiogenic shock within six hours, and 75\% develop it within 24 hours [3]. The use of digitalis drugs in the treatment of cardiogenic shock is controversial. When AMI is complicated by cardiogenic shock, myocardium in the ischemic area does not bind well with digitalis, thus increasing its toxicity, suggesting it should be avoided [2, 4].

The drug of choice for the treatment of cardiogenic shock is controversial. Milrinone has been shown to affect long-term mortality from cardiogenic shock [5], and levosimendan and adrenaline have been shown to have adverse side effects, which increase potential risks and incidence of adverse reactions $[6,7]$. Dobutamine has been shown to adversely increase the heart rate $[8,9]$ and yet is recommended by others with half of clinicians using it for treatment of cardiogenic shock $[10,11]$. The purpose of this study was to conduct a network meta-analysis on the clinical effects of medications used for the treatment of cardiogenic shock.

\section{Materials and Methods}

2.1. Literature Review. PubMed, EMBASE, Cochrane library, China National Knowledge Infrastructure (CNKI), Wanfang database, and Weipu database were searched for articles in Chinese or English using keywords Dopamine, Dobutamine, Epinephrine, Adrenaline, Norepinephrine, Noradrenaline, Milrinone, Natriuretic peptide, Recombinant human brain natriuretic peptide, Levosimendan, Cardiac shock, and Cardiogenic shock from January 1, 2009, to December 31, 2019. 
2.2. Inclusion and Exclusion Criteria. Articles meeting the following criteria were included: (1) randomized clinical trials related to cardiogenic shock drug therapy, (2) diagnosis of cardiogenic shock as described in the 2014 Chinese Heart Failure Guide [12], (3) cardiogenic shock as the main treatment target in the study, and (4) outcome indicators being mortality and adverse reactions. Studies were excluded if they met the following criteria: (1) nonexperimental studies such as "reviews" and "case reports," (2) contained duplicate or low quality data or insufficient information and clinical data, (3) literature on traditional Chinese medicine and proprietary Chinese medicines for cardiogenic shock, and (4) animal experiments.

\subsection{Data Extraction and Literature Quality Evaluation.} Extracted data included the author, publication date, average age, research method, sample number, mortality rate, and incidence of adverse reactions. We evaluated bias based on evaluation criteria from the Cochrane Handbook for Systematic Reviews of Interventions including random sequence generation, whether to hide the allocation scheme, whether to use blind method, completeness of the outcome data, whether to selectively report the research results, and other sources of bias. According to the Cochrane Handbook evaluation standards, the literature is divided into 3 levels: low deviation: all meet the Cochrane Handbook evaluation standard; medium deviation: 1 undescribed Cochrane Handbook evaluation standard; high deviation: there are 2 or more items not described or 1 item does not meet the Cochrane Handbook evaluation standard.

2.4. Statistical Processing Methods. The network metaanalysis was conducted using ADDIS 1. 16. 8. Data were first tested for consistency using a node-split model. Where there was no statistical difference between direct and indirect comparison $(P>0.05)$, the consistency model was used. Where there was a difference, an inconsistency model was used. The stability of the analysis results of the consistency model was tested using the inconsistency model. When the inconsistency factors included 0 and the inconsistency standard deviation included 1 and the consistency model results were more stable and reliable. Various analysis models were automatically iterated based on preset parameters, and the convergence of the iterative effect was judged by potential scale reduced factor (PSRF). When the PSRF value was close to or equal to $1(1 \leq \mathrm{PSRRF} \leq 1.05)$, the convergence is felt to be complete, and the model is believed to have good stability, rendering the analysis conclusion more reliable. Stata 14.0 was used to create the network diagram, and funnel diagrams were made to evaluate whether the included studies had publication bias.

\section{Results}

3.1. Literature Review. The included search terms identified 1387 articles. Using inclusion and exclusion criteria while evaluating the title, abstract, and full text of the literature, 28 articles were included, describing 1806 patients (Figure 1).

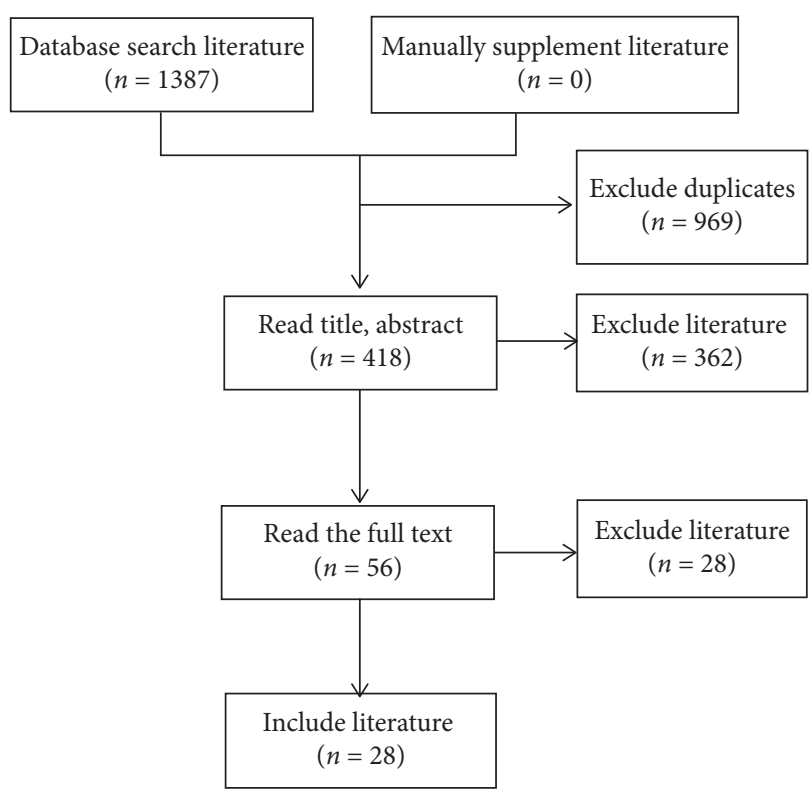

FIgURE 1: Literature screening process.

3.2. Basic Characteristics of the Literature. The 28 clinical studies [13-40] included were all clinical trials using medications to treat cardiogenic shock. Seven (25\%) of the 28 studies were conducted before 2015, and the remaining 21 (75\%) studies were concentrated after 2015. The treatment cycle and dosage of the drugs in each group were basically the same, and the difference was not statistically significant (Table 1).

3.3. Evaluation of Included Studies. Of the 28 studies [13-40], 7 (25\%) clearly stated the method of randomization (random number table, admission order, etc.), 1 (3.5\%) of the studies described the allocation concealment method, and none described the method of blinding; other sources of bias were unknown; the baseline patient characteristics of the studies were basically the same (Table 2).

\subsection{Network Meta-Analysis Results}

3.4.1. Network Diagram of Included Interventions. Each dot in the network diagram represents a drug, and a wired segment directly connected between the two points indicates a direct comparison between the two drugs. The larger the dot, the higher the frequency of study drugs being included in the reticulation analysis. The wider the line between the two dots, the higher the frequency of comparisons between drugs (Figures 2 and 3).

3.4.2. Node-Split Model Test and Convergence Judgment. Case fatality rate and incidence of adverse reactions were evaluated by node-split model method. Both $P$ values were greater than 0.05 , suggesting that there was no statistical inconsistency, supporting use of the consistency model for analysis. Both the consistency model analysis and the 


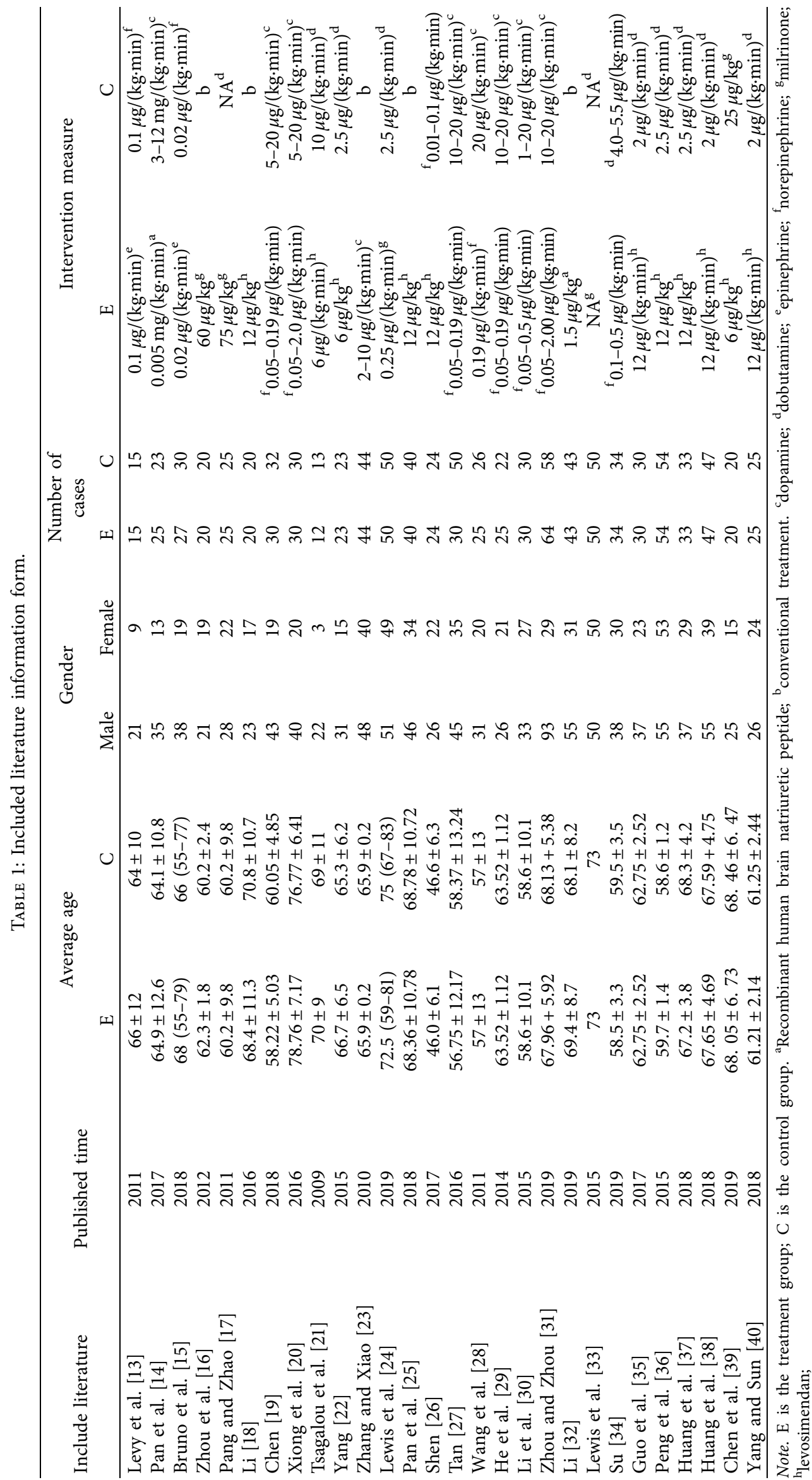


TABLE 2: Literature bias risk assessment results.

\begin{tabular}{|c|c|c|c|c|c|c|}
\hline $\begin{array}{l}\text { Include } \\
\text { literature }\end{array}$ & Stochastic method & $\begin{array}{c}\text { Allocation } \\
\text { concealment }\end{array}$ & $\begin{array}{l}\text { Blind } \\
\text { method }\end{array}$ & Outcome data integrity & $\begin{array}{l}\text { Selective report } \\
\text { results }\end{array}$ & $\begin{array}{c}\text { Other sources of } \\
\text { bias }\end{array}$ \\
\hline Levy et al. [13] & $\begin{array}{l}\text { Randomize the } \\
\text { code }\end{array}$ & Unclear & Unclear & Not lost to follow-up & No & Unclear \\
\hline Pan et al. [14] & Unclear & Label & Unclear & $\begin{array}{c}\text { Lost to follow-up, ITT } \\
\text { analysis }\end{array}$ & No & Unclear \\
\hline Bruno et al. [15] & Unclear & Unclear & Unclear & $\begin{array}{l}\text { Lost to follow-up, ITT } \\
\text { analysis }\end{array}$ & No & Unclear \\
\hline Zhou et al. [16] & Admission order & Unclear & Unclear & $\begin{array}{c}\text { Lost to follow-up, ITT } \\
\text { analysis }\end{array}$ & No & Unclear \\
\hline $\begin{array}{l}\text { Pang and Zhao } \\
{[17]}\end{array}$ & Unclear & Unclear & Unclear & Not lost to follow-up & No & Unclear \\
\hline $\mathrm{Li}[18]$ & Unclear & Unclear & Unclear & Not lost to follow-up & No & Unclear \\
\hline Chen [19] & Unclear & Unclear & Unclear & Not lost to follow-up & No & Unclear \\
\hline Xiong et al. [20] & Unclear & Unclear & Unclear & Not lost to follow-up & No & Unclear \\
\hline $\begin{array}{l}\text { Tsagalou et al. } \\
\text { [21] }\end{array}$ & Unclear & Unclear & Unclear & Not lost to follow-up & No & Unclear \\
\hline Yang [22] & Unclear & Unclear & Unclear & Not lost to follow-up & No & Unclear \\
\hline $\begin{array}{l}\text { Zhang and Xiao } \\
\text { [23] }\end{array}$ & Unclear & Unclear & Unclear & Not lost to follow-up & No & Unclear \\
\hline Lewis et al. [24] & Unclear & Unclear & Unclear & Not lost to follow-up & No & Unclear \\
\hline Pan et al. [25] & Unclear & Unclear & Unclear & Not lost to follow-up & No & Unclear \\
\hline Shen [26] & Unclear & Unclear & Unclear & $\begin{array}{c}\text { Lost to follow-up, ITT } \\
\text { analysis }\end{array}$ & No & Unclear \\
\hline Tan [27] & Unclear & Unclear & Unclear & Not lost to follow-up & No & Unclear \\
\hline Wang et al. [28] & Unclear & Unclear & Unclear & Not lost to follow-up & No & Unclear \\
\hline He et al. [29] & Unclear & Unclear & Unclear & Not lost to follow-up & No & Unclear \\
\hline Li et al. [30] & Unclear & Unclear & Unclear & $\begin{array}{l}\text { Lost to follow-up, ITT } \\
\text { analysis }\end{array}$ & No & Unclear \\
\hline $\begin{array}{l}\text { Zhou and Zhou } \\
\text { [31] }\end{array}$ & Unclear & Unclear & Unclear & Not lost to follow-up & No & Unclear \\
\hline $\operatorname{Li}[32]$ & $\begin{array}{c}\text { Random number } \\
\text { table }\end{array}$ & Unclear & Unclear & Not lost to follow-up & No & Unclear \\
\hline Lewis et al. [33] & Unclear & Unclear & Unclear & Not lost to follow-up & No & Unclear \\
\hline $\mathrm{Su}[34]$ & Random grouping & Unclear & Unclear & Not lost to follow-up & No & Unclear \\
\hline Guo et al. [35] & Random number & Unclear & Unclear & Not lost to follow-up & No & Unclear \\
\hline Peng et al. [36] & Unclear & Unclear & Unclear & Not lost to follow-up & No & Unclear \\
\hline Huang et al. [37] & $\begin{array}{l}\text { Random number } \\
\text { table }\end{array}$ & Unclear & Unclear & Not lost to follow-up & No & Unclear \\
\hline Huang et al. [38] & Unclear & Unclear & Unclear & Not lost to follow-up & No & Unclear \\
\hline Chen et al. [39] & $\begin{array}{l}\text { Random number } \\
\text { table }\end{array}$ & Unclear & Unclear & Not lost to follow-up & No & Unclear \\
\hline $\begin{array}{l}\text { Yang and Sun } \\
{[40]}\end{array}$ & Unclear & Unclear & Unclear & Not lost to follow-up & No & Unclear \\
\hline
\end{tabular}

inconsistency model test of the network meta-analysis have PSRF values between 1 and 1.05, indicating that the convergence is good and the results are stable.

3.4.3. Network Meta-Analysis of Case Fatality Rate under the Consistency Model. Twenty of the 1199 studies [13-32] used case fatality rate as the outcome indicator, and these were included in a network meta-analysis. According to the ranking probability map of treatment measures (Rank 8 being the best and Rank 1 being the worst), the ability of drugs to reduce fatality was as follows: milrinone $>$ levosimendan $>$ norepinephrine $>$ recombinant human brain natriuretic peptide $>$ dobutamine $>$ epinephrine $>$ dopamine $>$ conventional treatment.
Milrinone appeared to be the best treatment option to reduce the case fatality rate (with a probability of $44 \%$ ), with levosimendan coming in second with a probability of $26 \%$.

3.4.4. Network Meta-Analysis of the Incidence of Adverse Reactions under the Consistency Model. Eighteen of 1317 studies [24-40] used incidence of adverse reactions as the outcome indicator, and these were included in a network metaanalysis. According to the ranking probability map of treatment measures, the side effect profile from best to worst was as follows: milrinone $>$ recombinant human brain natriuretic peptide $>$ norepinephrine $>$ levosimendan $>$ conventional treatment $>$ epinephrine $>$ dobutamine $>$ dopamine. Milrinone appeared to be associated with the least amount of adverse 


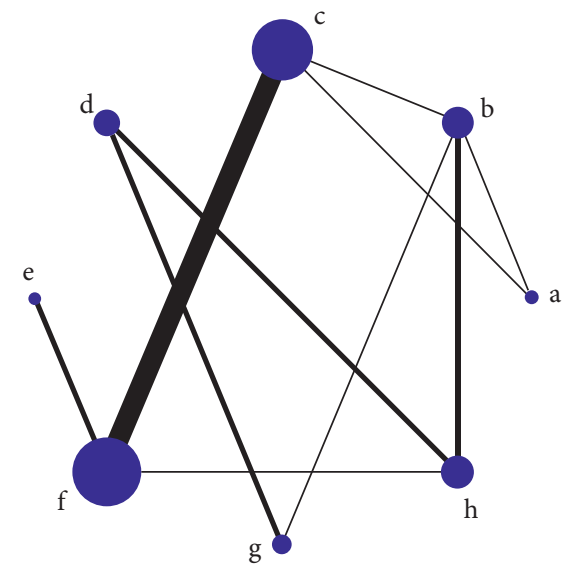

Figure 2: Network diagram of case fatality rate. Note: a, recombinant human brain natriuretic peptide; $b$, conventional treatment; $c$, dopamine; $d$, dobutamine; e, epinephrine; f, norepinephrine; g, milrinone; $\mathrm{h}$, levosimendan.

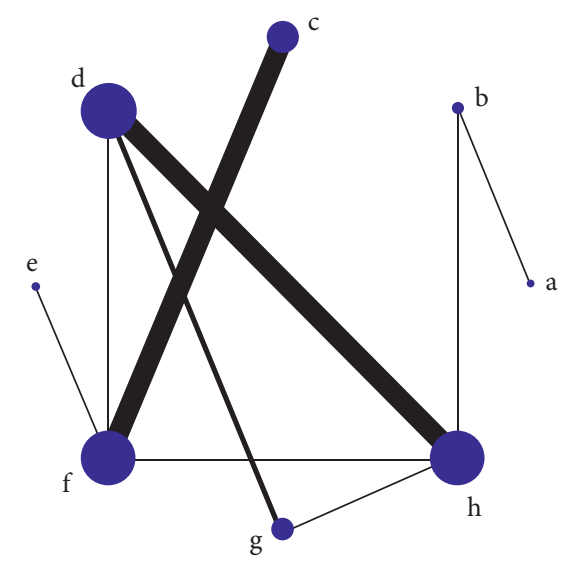

Figure 3: Network diagram of incidence of adverse reactions. Note: $a$, recombinant human brain natriuretic peptide; $b$, conventional treatment; $c$, dopamine; $d$, dobutamine; e, epinephrine; $f$, norepinephrine; g, milrinone; h, levosimendan.

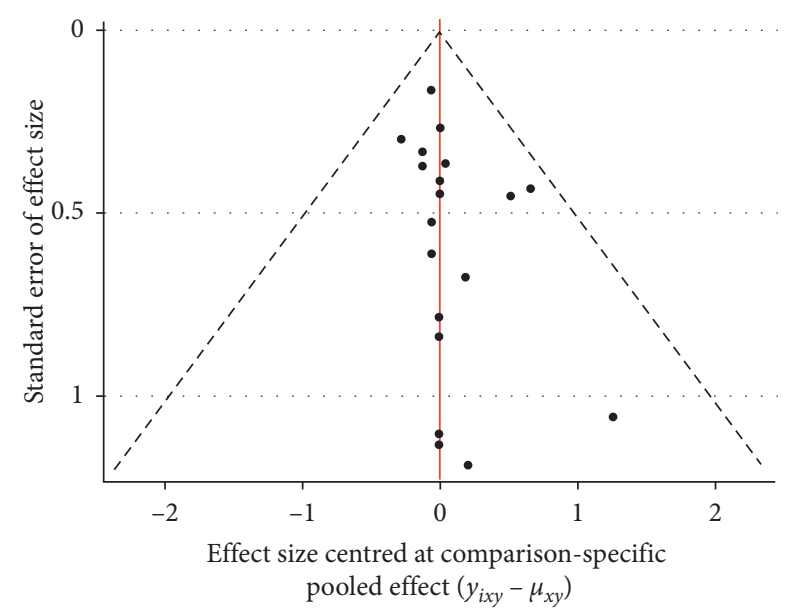

FIGURE 4: Funnel plot of case fatality rate for outcome indicator.

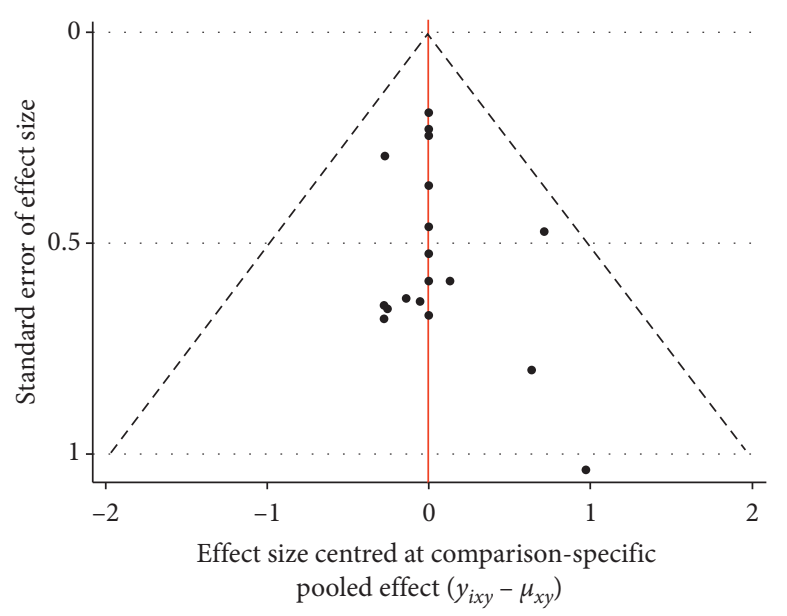

FIGURE 5: Funnel plot of incidence of adverse reactions for outcome indicators.

reactions (with a probability of 55\%), with recombinant human brain natriuretic peptide coming in second (with a probability of $32 \%)$.

3.4.5. Inconsistency Model Testing. The consistency model of the two outcome indicators was analyzed and inconsistency models were used to test the stability of the results. Results demonstrated that the inconsistency factors all included 0 and the inconsistency standard deviations all included 1 . This means that the results of the consistency model were stable and reliable.

3.5. Publication Bias. Funnel plots were created to identify small sample effects in the analysis. Funnel plots were created for the two outcome indicators for publication bias testing. Results demonstrated that the included studies were roughly symmetrically distributed on both sides of the funnel plot, and therefore the possibility of publication bias was felt to be small (Figures 4 and 5).

\section{Discussion}

Cardiogenic shock is a serious disease that, if not treated expeditiously and appropriately at an early stage, can have a high risk of mortality. The pathological changes of cardiogenic shock usually include two parts: one is abnormal hemodynamics and the other is insufficient perfusion of surrounding tissues. The prognosis of patients is closely related to the degree of hemodynamic abnormalities, so the rapid correction of hemodynamic abnormalities in patients with cardiogenic shock is the key to treating cardiogenic shock [41]. In clinical treatment of cardiogenic shock, blood volume is usually appropriately supplemented, and positive inotropic drugs combined with vasoactive drugs are used. For example, calcium sensitizers, levosimendan, can be combined with troponin to enhance myocardial contractility, expand coronary arteries, and improve myocardial ischemia; $\beta$-receptor agonist dobutamine mainly stimulates myocardial $\beta 1$ receptors and produces a positive inotropic effect. Phosphodiesterase inhibitors milrinone can inhibit 
phosphodiesterase III, increase the content of cyclic adenosine monophosphate in myocardial cells [42], exert positive inotropic effects, expand blood vessels, and improve hemodynamics. Recombinant human brain natriuretic peptide with peripheral vasodilators has similar biological activity with human-derived BNP, which can dilate blood vessels, reduce heart load, and inhibit ventricular remodeling. Although many medications have been investigated for the treatment of cardiogenic shock, a direct comparison of the effectiveness of these medications has not previously been conducted. We aimed to compare previously described medications for use in cardiogenic shock with the hope of identifying preferable medications that could be administered quickly in an emergency setting.

Our study identified milrinone as being the most effective medication for reducing fatality and having the best side effect profile. Milrinone can improve the patient's hemodynamic abnormalities and hypoperfusion status, thereby rapidly improving cardiac function and correcting heart failure, Therefore, the rapid and effective application of milrinone is of great significance to save patients' lives. Levosimendan and recombinant human brain natriuretic peptide were the second most effective drugs in reducing the case fatality rate and adverse reactions. They have a positive effect on improving the clinical symptoms and prognosis of patients, and they can be used according to the patient's condition.

\section{Limitations}

Limitations of this study include lesser quality of some studies included in the analysis, small sample sizes in others, and varied lengths of treatment across studies. These factors could affect the reliability of the reticulated meta-analysis. It is hoped that larger, multicenter randomized controlled trials will provide clinical data in the future to achieve a more comprehensive understanding and evaluation.

\section{Conclusion}

In this paper, a reticular meta-analysis system is used to evaluate the difference in the efficacy of various drugs on different outcome indicators, which provides evidencebased evidence for clinical treatment, is conducive to more effective control of clinical symptoms and disease progression, and also for further clinical trials provided a reference. The analysis results showed that milrinone had the best effect in reducing the case fatality rate and the incidence of adverse reactions in patients with cardiogenic shock. Levosimendan and recombinant human brain natriuretic peptide were the second most effective drugs in reducing the case fatality rate and adverse reactions. Milrinone can improve the patient's hemodynamic abnormalities and hypoperfusion status and has the best clinical effect to reduce the patient's case fatality rate and incidence of adverse reactions. Therefore, milrinone is recommended as the clinically preferred drug for cardiogenic shock.

\section{Data Availability}

The data used in the article come from clinical research, and the data used in the article can be obtained from PubMed, EMBASE, Cochrane library, China National Knowledge Infrastructure (CNKI), Wanfang database, and Weipu database. The data used to support the findings of this study are included within the supplementary information files.

\section{Conflicts of Interest}

The authors declare that there are no conflicts of interest regarding the publication of this paper.

\section{Authors' Contributions}

Xianyong Liao, Lin Qian, and Song Zhang contributed equally to this work.

\section{Supplementary Materials}

The [zip] data used to support the findings of this study are included within the supplementary information files. (Supplementary Materials)

\section{References}

[1] W. Chen, L. Zhao, L. Li et al., "Predictive value of extravascular pulmonary water index and cardiac index on the prognosis of patients with cardiogenic shock," Chinese Journal of Circulation, vol. 37, no. 11, pp. 895-898, 2014.

[2] S. Zhang, "Progress in diagnosis and treatment of cardiogenic shock and interpretation of guidelines," Journal of Medical Research, vol. 46, no. 478, pp. 7-9, 2017.

[3] K. Werdan, M. Russ, M. Buerke et al., "Evidence-based management of cardiogenic shock after acute myocardial infarction," Interventional Cardiology Review, vol. 8, no. 2, pp. 73-80, 2013.

[4] C. Huang, "Guidelines for cardiovascular emergency management-acute myocardial infarction complicated with cardiogenic shock," Chinese Journal of Practical Internal Medicine, vol. 20, no. 9, pp. 515-517, 2000.

[5] S. Jing, X. Zhang, and J. Lu, "Efficacy of milrinone in the treatment of heart failure after acute myocardial infarction," Journal of Kunming Medical College, vol. 31, no. 8, pp. 134136, 2010.

[6] X. Lian, "Clinical observation of dopamine combined with norepinephrine in the treatment of cardiogenic shock," Northern Medicine, vol. 14, no. 4, p. 43, 2017.

[7] C. L. Erley, "Epinephrine versus norepinephrine for cardiogenic shock after acute myocardial infarction," The Journal of Emergency Medicine, vol. 55, no. 5, p. 736, 2018.

[8] T. Hashim, K. Sanam, M. Revilla-Martinez et al., "Clinical characteristics and outcomes of intravenous inotropic therapy in advanced heart failure," Circulation: Heart Failure, vol. 8, no. 5, pp. 880-886, 2015.

[9] S. Tariq and W. Aronow, "Use of inotropic agents in treatment of systolic heart failure," International Journal of Molecular Sciences, vol. 16, no. 12, pp. 29060-29068, 2015.

[10] S. Champion and N. Deye, "Management of cardiogenic shock: results from a survey in France and Belgium," Annales de cardiologie et d'angeiologie, vol. 66, no. 2, 2017. 
[11] A. Mebazaa, R. Bellomo, R. Pirracchio et al., "Epinephrine and short-term survival in cardiogenic shock: an individual data meta-analysis of 2583 patients," Intensive Care Medicine, vol. 44, no. 6, pp. 847-856, 2018.

[12] Chinese Medical Association Cardiovascular Disease Branch, "Chinese Journal of Cardiovascular Disease Editorial Board. Chinese heart failure diagnosis and treatment guide 2014," Chinese Journal of Cardiovascular Disease, vol. 42, no. 2, pp. 98-122, 2014

[13] B. Levy, P. Perez, J. Perny, C. Thivilier, and A. Gerard, "Comparison of norepinephrine-dobutamine to epinephrine for hemodynamics, lactate metabolism, and organ function variables in cardiogenic shock. A prospective, randomized pilot study," Critical Care Medicine, vol. 39, no. 3, pp. 450455, 2011.

[14] Y. Pan, Z. Lu, J. Hang et al., "Effects of low-dose recombinant human brain natriuretic peptide on anterior myocardial infarction complicated by cardiogenic shock," Brazilian Journal of Cardiovascular Surgery, vol. 32, no. 2, pp. 96-103, 2017.

[15] L. Bruno, R. Clere-Jehl, L. Annick et al., "Epinephrine versus norepinephrine for cardiogenic shock after acute myocardial infarction," Journal of the American College of Cardiology, vol. 72, no. 2, 2018.

[16] D. Zhou, L. Wang, Q. Wang et al., "Efficacy of nitroglycerin combined with milrinone in the treatment of acute myocardial infarction-induced cardiogenic shock," Clinical Medical Research, vol. 29, no. 3, pp. 421-422, 2012.

[17] Z. Pang and W. Zhao, "Clinical observation of milrinone in treating cardiogenic shock induced by acute myocardial infarction," Journal of Practical Clinical Medicine, vol. 15, no. 15, pp. 82-83, 2011.

[18] C. Li, PICCO Monitoring of Levosimendan in Patients with Acute Myocardial Infarction Complicated with Cardiogenic Shock, The Chinese People's Liberation Army Medical College, Beijing China, 2016.

[19] X. Chen, "Dynamic blood lactate assay to evaluate the clinical efficacy of norepinephrine and dopamine in the treatment of cardiogenic shock," Liu Gang Technology, no. 1, pp. 53-55, 2018.

[20] R. Xiong, Yu Zhou, J. Sun et al., "Efficacy of dopamine and norepinephrine in patients with cardiogenic shock," Chinese Journal of Elderly Multiple Organ Diseases, vol. 15, no. 12, pp. 919-921, 2016.

[21] E. Tsagalou, J. Kanakakis, M. Anastasiou-Nana et al., "Hemodynamic effects of levosimendan in acute myocardial infarction complicated by cardiogenic shock and high systemic vascular resistance," Acute Cardiac Care, vol. 11, no. 2, pp. 99-106, 2009.

[22] H. Yang, "Efficacy of levosimendan in the treatment of cardiogenic shock," Strait Pharmaceutical Journal, vol. 26, no. 9, pp. 105-107, 2014.

[23] G. Zhang and C. Xiao, "Efficacy of dopamine combined with levocarnitine in the treatment of acute myocardial infarction with cardiogenic shock," Journal of Guangdong Medical College, vol. 28, no. 4, pp. 413-414, 2010.

[24] T. C. Lewis, C. Aberle, D. Altshuler, G. L. Piper, and J. Papadopoulos, "Comparative effectiveness and safety between milrinone or dobutamine as initial inotrope therapy in cardiogenic shock," Journal of Cardiovascular Pharmacology and Therapeutics, vol. 24, no. 2, pp. 130-138, 2019.

[25] X. Pan, Y. Chen, and Q. Fang, "Application of pulse indication continuous cardiac output monitoring of levosimendan in elderly patients with acute myocardial infarction and cardiogenic shock," Chinese Journal of Gerontology, vol. 38, no. 3, pp. 519-521, 2018.

[26] X. Shen, "Clinical observation of levosimendan combined with norepinephrine in the treatment of cardiogenic shock," Health Wenhui, no. 9, pp. 20-21, 2017.

[27] Z. Tan, "Comparison of the application of dopamine and norepinephrine in cardiogenic shock," Modern Diagnosis and Treatment, vol. 27, no. 1, pp. 67-68, 2016.

[28] G. Wang, Y. Guo, J. Hou et al., "Comparison of curative effect of dopamine and norepinephrine on cardiogenic shock," China Practical Medical Journal, vol. 38, no. 2, pp. 75-76, 2011.

[29] Y. He, C. Lu, Y. Jiao et al., "Comparison of clinical efficacy of dopamine and norepinephrine in the treatment of cardiogenic shock," Northern Pharmaceutical, vol. 11, no. 2, p. 5151, 2014.

[30] J. Li, L. Liu, M. Guo et al., "Effects of norepinephrine and dopamine on hemodynamics and tissue perfusion oxygen metabolism in patients with cardiogenic shock," Medical Theory and Practice, vol. 28, no. 23, pp. 3171-3173, 2015.

[31] Y. Zhou and B. Zhou, "Application of norepinephrine in cardiogenic shock," Laboratory Medicine and Clinic, vol. 16, no. 8, pp. 1092-1096, 2019.

[32] R. Li, "Efficacy and prognosis of recombinant human brain natriuretic peptide on acute myocardial infarction and cardiogenic shock," Anhui Medical and Pharmaceutical Journal, vol. 23, no. 4, pp. 696-699, 2019.

[33] T. Lewis, C. Aberle, D. Esaian, and J. Papadopoulos, "Efficacy and safety of milrinone versus dobutamine in cardiogenic shock," Critical Care Medicine, vol. 43, no. 12 Suppl 1, p. 34, 2015.

[34] H. Su, "Clinical effect of norepinephrine combined with dobutamine on cardiogenic shock," China Contemporary Medicine, vol. 26, no. 12, pp. 131-134, 2019.

[35] Q. Guo, W. Song, R. Cui et al., "Clinical study on the treatment of cardiogenic shock after myocardial infarction with levosimendan," Modern Drug and Clinical Medicine, vol. 32, no. 6, pp. 1029-1033, 2017.

[36] L. Peng, L. Rui, Y. Zou et al., "Efficacy evaluation of levosimendan in treating 54 cases of acute myocardial infarction complicated with cardiogenic shock," China Pharmaceutical Industry, vol. 24, no. 17, pp. 46-47, 2015.

[37] J. Huang, X. Hu, and J. Xie, "Clinical curative effect of levosimendan injection in treating cardiogenic shock after myocardial infarction," China Healthcare Nutrition, vol. 28, no. 24 , p. $222,2018$.

[38] M. Huang, W. Chen, T. Lin et al., "Evaluation of the effect of levosimendan on cardiogenic shock after myocardial infarction. Knowledge on prevention and treatment of cardiovascular diseases," Academic Edition, no. 11, pp. 38-40, 2018.

[39] C. Xu, Z. Duan, and Z. Liu, "Clinical observation of levosimendan and milrinone on patients with low cardiac function during cardiac surgery," Modern Hospital, vol. 19, no. 2, 2019.

[40] J. Yang and H. Sun, "Clinical analysis of levosimendan injection for cardiogenic shock after myocardial infarction," Health Weekly, no. 16, pp. 18-19, 2018.

[41] C. Huang and H. Xia, "Management principles and experience of acute myocardial infarction with cardiogenic shock," Chinese Journal of Practical Internal Medicine, vol. 27, no. 2, pp. 95-97, 2007.

[42] X. Ma, "Clinical observation of milrinone in the treatment of congestive heart failure," Journal of Practical Clinical Medicine, vol. 15, no. 5, p. 76, 2011. 